\title{
Improved image reconstruction based on ultrasonic transmitted wave computerized tomography on concrete
}

\author{
Honghui Fan and Hongjin Zhu*
}

\begin{abstract}
Considering the non-uniqueness and instability of concrete defect detection in construction engineering, ultrasonic time of flight data and maximum likelihood expectation maximization algorithm were proposed to improve the readability of the ultrasonic reconstruction image. Ultrasonic transmitted waves reflect internal information when through concrete structures. Measurement signals were regularized and processed as transit time parameters. Maximum likelihood expectation maximization algorithm was optimized for image reconstruction of the concrete structure. The interpolation data as the density of measurement data was used to improve the readability of reconstruction images. The experimental measurement system, improved image reconstruction algorithm, and interpolation method were verified in detail by using simulation and concrete phantoms. The proposed image reconstruction technique based on maximum likelihood expectation maximization overcame the disadvantages of the traditional image reconstruction method, and it was effective to improve the accuracy and the quality of image reconstruction on concrete. Time of flight data and normalized interpolation were discussed in detail during image reconstruction. The results showed that it could make the position of an abnormal object more obvious, and it was more accurate for reflecting the internal structure of concrete.
\end{abstract}

Keywords: Computerized tomography, Ultrasonic transmitted wave, Image reconstruction, Maximum likelihood expectation maximization, Transit time parameters

\section{Introduction}

The ultrasonic computerized tomography technique is used to detect internal defects of non-transparent objects $[1,2]$. There are many research reports on ultrasonic computerized tomography systems for non-destructive inspection to find hidden defects or quality problems [3]. Computed tomography ultrasound was based on the estimated spatial distribution of the ultrasonic velocity to achieve detection $[4,5]$. Transit time parameter ultrasonic testing was based on the velocity distribution of concrete to detect internal cavity, cracks, and defects $[6,7]$. Yanagida found that the computed tomography images of wood based on filtered back projection image reconstruction system in their report. It was modified to reconstruct images using the incomplete transit time parameters [8]. The filtered back projection (FBP) algorithm has been

\footnotetext{
* Correspondence: zhuhongjin@jsut.edu.cn

School of Computer Engineering, Jiangsu University of Technology, Changzhou 213001, Jiangsu, China
}

\section{Springer Open}

(c) The Author(s). 2018 Open Access This article is distributed under the terms of the Creative Commons Attribution 4.0 International License (http://creativecommons.org/licenses/by/4.0/), which permits unrestricted use, distribution, and reproduction in any medium, provided you give appropriate credit to the original author(s) and the source, provide a link to the Creative Commons license, and indicate if changes were made. some research reports, they were extended to the fanbeam data acquisition geometry $[9,10]$. In image reconstruction, there were some negative values of pixels with FBP algorithm [11]. It was difficult to place the number of fixed pixels as FBP reconstruction image of the distribution of the decay level and presumption [12,13].

Many researchers have conducted research on concrete based on ultrasonic signal detection, image reconstruction algorithm, and measurement system design. Akamatsu proposed an optimization scheme for the filter function in re-image reconstruction [14]. Jung overcame the Gibbs phenomenon and improved the reconstructed image quality [15]. Chandra introduced the finite-angle iterative image reconstruction of model fusion, which effectively preserves the original target features and improves the quality of small-angle projection data reconstruction [16]. Muldoon et al. of the University of Edinburgh improved the FBP ultrasonic 
tomography method to identify the hole defects in the grouting pipe of the post-tensioned prestressed reinforced concrete beam and verified the test [17]. Yan et al. have combined research on hash changes, many-core processors, and image processing [18-20], and some researchers had researched the understanding of image reconstruction results and knowledge rebellion [21, 22].

The maximum likelihood expectation maximization (MLEM) algorithm was used widely in medical computed tomography [23, 24]. It has a few advantages when compared to FBP in the image reconstruction process $[25,26]$. MLEM algorithm in image reconstruction process has advantages: correct absorption, scattering, resolution, and reconstruction pixel values are all positive [27, 28]. Some research considerations are relying on MLEM algorithm to improve the image quality of anisotropic acoustic characteristics and time of flight (TOF) data interpolation, which could detect internal defects of an object $[29,30]$.

Because of design, construction, and materials, the concrete may have defects such as segregation, holes, honeycomb, and low density during pouring. The quantitative detection of the internal structure of concrete becomes relatively difficult due to its hard integrity and complex physical properties after concrete infusion. Although $\mathrm{X}$-ray tomography could analyze the internal structures such as holes and steel bars in concrete quickly and accurately, it is difficult to popularize in engineering the quality inspection because of its radioactive characteristics. The research data show that the detection of concrete based on ultrasonic is still in the qualitative or semi-quantitative stage. Some researches are based on the study of ultrasonic signals and inversion image reconstruction algorithms for the tomography of concrete. The effect of the inherent physical properties of the concrete on the ultrasonic propagation signal is not fully considered.

In this paper, we proposed an inspection system for concrete by using ultrasonic transmitted waves based on the modified ultrasound propagation path and using the MLEM algorithm for image reconstruction. The main content of the research is to construct a linear interpolation system for global transit time parameters and solve the problem of a large amount of data required for image construction based on the iterative reconstruction algorithm of ordered subset maximum expectation value. Depending on ultrasonic velocity and measurement path prediction, we calculated the correct path for the construction images of concrete. The relation between prediction wave paths and image reconstruction systems was examined in detail using simulation and concrete phantoms.

\section{Proposed methods}

\subsection{Image reconstruction algorithm}

The FBP algorithm is a spatial domain processing technique based on the Fourier transform theory. It is characterized by convolution processing of each projection under the projection angle before back projection, thereby improving the shape artifact caused by the point spread function, and the reconstructed image quality is better.

The data $I_{o}$ obtained from the observation is expressed as a function of the line integral for route $s$ physical weight distribution $f(x, y)$ of the measurement section (Eq. (1)). The specific principles and experiments are described in our previous research [31].

$$
I_{O}=\int_{s} f(x, y) d s
$$

The MLEM algorithm type of iterative reconstruction method is developed for CT reconstruction. The MLEM algorithm iteratively corrects the estimated slowness from using Eq. (2); the reasoning and composition of formulas have been explained in detail in our previous study. Here, $i$ is the index of a measurement pass, $j$ is the index of a pixel, $\lambda_{j}$ is the slowness at the $j$ th pixel, $y_{i}$ is the TOF data of the $i$ th pathway, and $C_{i j}$ is the contribution rate of the $j$ th pixel to the time of flight value obtained at the $i$ th pass.
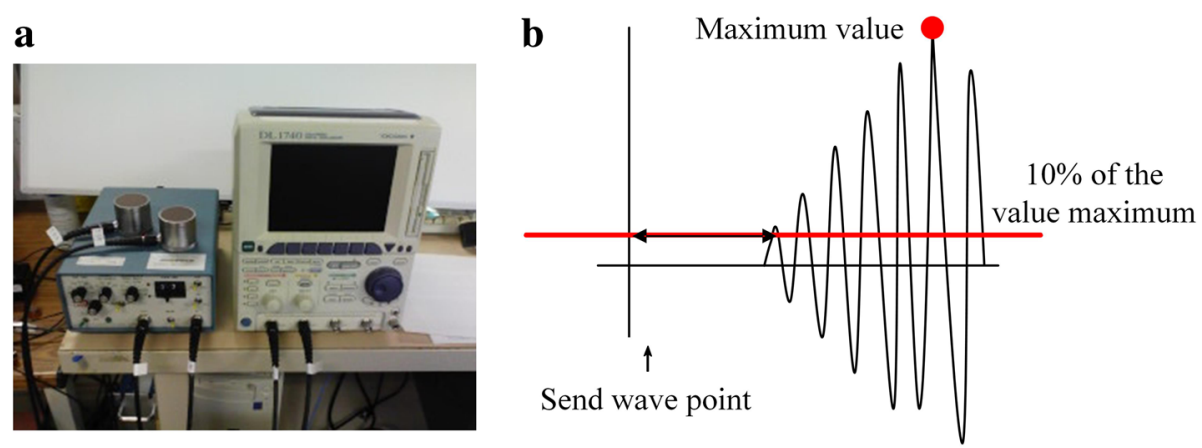

Fig. 1 Experimental system diagram: a measurement system, b Estimation of transit time parameters 

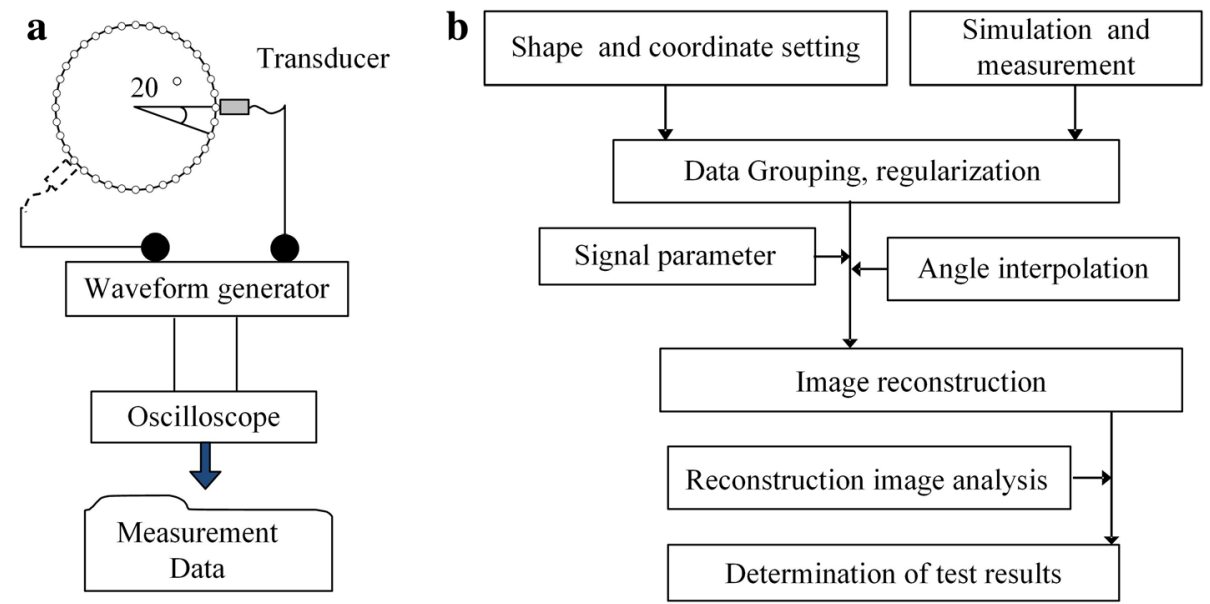

Fig. 2 Data and image reconstruction process: a data collection process, $\mathbf{b}$ flowchart for image reconstruction

$$
\lambda_{j}^{(k+1)}=\frac{\lambda_{j}^{(k)}}{\sum_{i=1}^{n} C_{i j}^{(k)}} \sum_{i=1}^{n} \frac{y_{i} C_{i j}^{(k)}}{\sum_{l=1}^{m} C_{i l}^{(k)} \lambda_{l}^{(k)}}
$$

\subsection{Experimental methods}

The measurement system was presented in Fig. 1a. The experimental system was composed of two transducers, a waveform generator and an oscilloscope. The two transducers were used respectively for transmitting and receiving ultrasonic signals. Figure $1 \mathrm{~b}$ showed an example of the flow of measurement when the measuring points were set to 36 on a circular cross-section periphery of the measuring object. The measurement points were placed on the measuring object based on a central angle of 10. In our system, TOF data measurement method was the similarity in the report which explained in Yanagida report. The data could be collected for ultrasonic wave propagation time between the measurement points of the total 306 pairs by repeating the same measurement. The transit time parameters were calculated based on the received waveform of the collected data. The propagation time was the first time signal to reach the threshold which is shown in Fig. 1b, and the signal was the interval from the implanted transmission to the point. In the conventional method, it was laced at $10 \%$ of the amplitude maximum amplitude of the received waveform with the threshold uniformly. The level of the received waveform caused by the ultrasonic attenuation could be measured with no change in the transit time parameters. After such treatment, the relative transit time parameters could be calculated.

The iterative algorithm based on the ordered subset maximum expectation requires a large number of imaging parameters. In order to ensure that the reconstructed image can accurately reveal the internal structure of the concrete, the measurement path is required to cover the measured fault plane as comprehensively as possible. In our test system, 36 equal measuring points are set on the

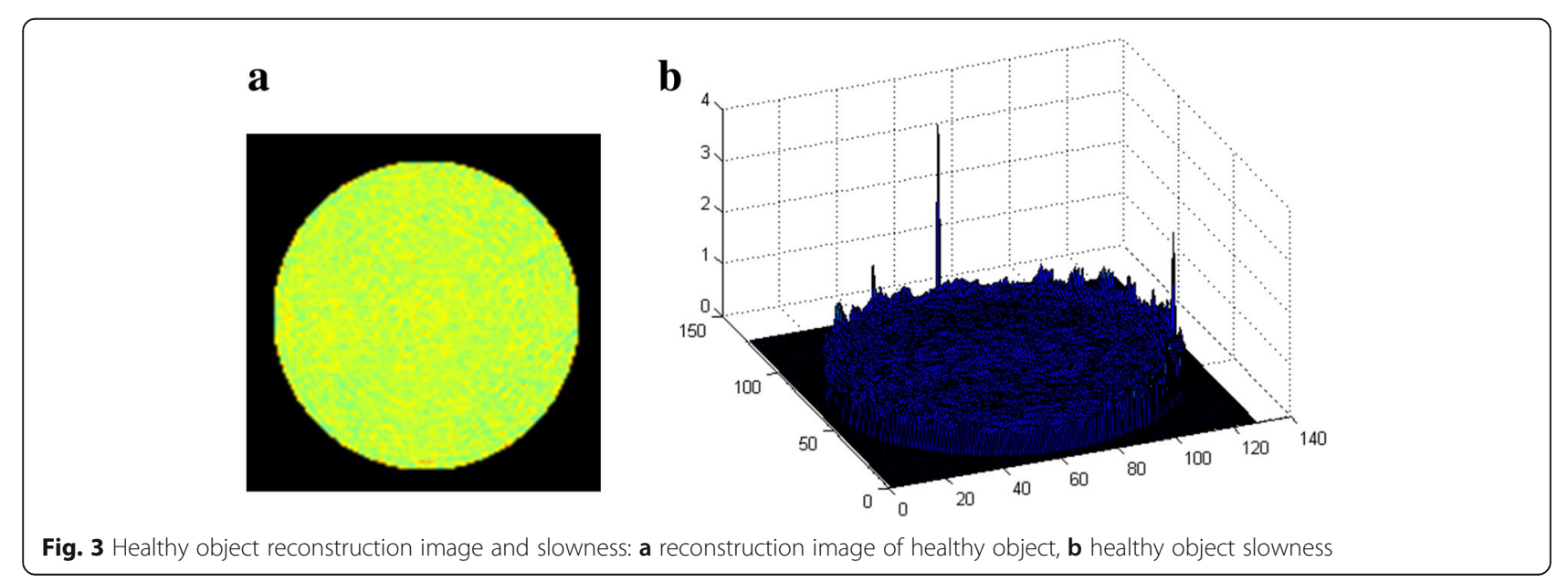



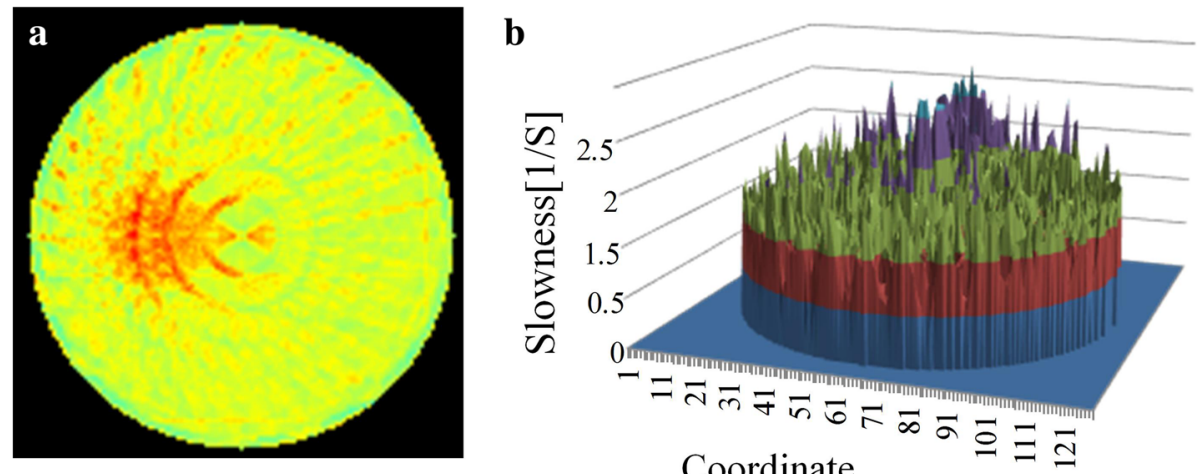

Fig. 4 Three hundred six data reconstruction image and slowness: a reconstruction image with 306 data, b slowness of reconstruction image with 306 data

concrete and measured from 20 to $180^{\circ}$ point by point according to the central angle of the signal transmitting point and the signal receiving point.

Because the transit signal was a parameter with the internal structure of the object, 36 measuring points were placed $10^{\circ}$ apart on the circumference of the test sample and labeled with numbers from 0 to 35 . There was an exchange when transmitting and receiving on the same measurement point. Therefore, all the measurement paths should be 306. Figure 2a showed the flow of measurement as an example when to set the measuring points at 36 on a circular cross-section periphery of the measuring object.

To obtain 306 transit time parameters, all measurement paths required time about $2 \mathrm{~h}$; the number and duration of manual measurement of data were considered the upper limit of space and time. Transit time parameters from the measurement wave were related projection data which were based on the sound velocity distribution required for image reconstruction. This transmission time had means that represented by the line integral of the distribution, and it was the speed inverse of sound along the propagation path. The process of image reconstruction based on MLEM in our system was shown in Fig. 2b.
However, since the amount of data was limited by the measurement time problem, it was necessary to compensate for the amount of data by interpolation. The transit time parameter profile of a fan beam shaped should be made according to the collected data of the measured total path. The large angle between the fan beams was used, and an interpolation process at regular intervals was performed to compensate for the roughness of the projection angle. After doing a parallel section and sorting by projection angle between parallel beams, interpolating transit time was used to compensate insufficient data. And the three beams' width was applied for a moving average filter for suppressing the high spatial frequency components.

In the system, when the ultrasonic wave was not received by the attenuation inside the measurement object, and if the path could not be measured for some reason, the data of that part would be supplemented by interpolation.

\section{Simulation experiment}

Concrete was composed of water, cement, sand, and other ingredients. The material constant in the case of the acoustic velocity of a healthy portion was $5000 \mathrm{~m} / \mathrm{s}$,
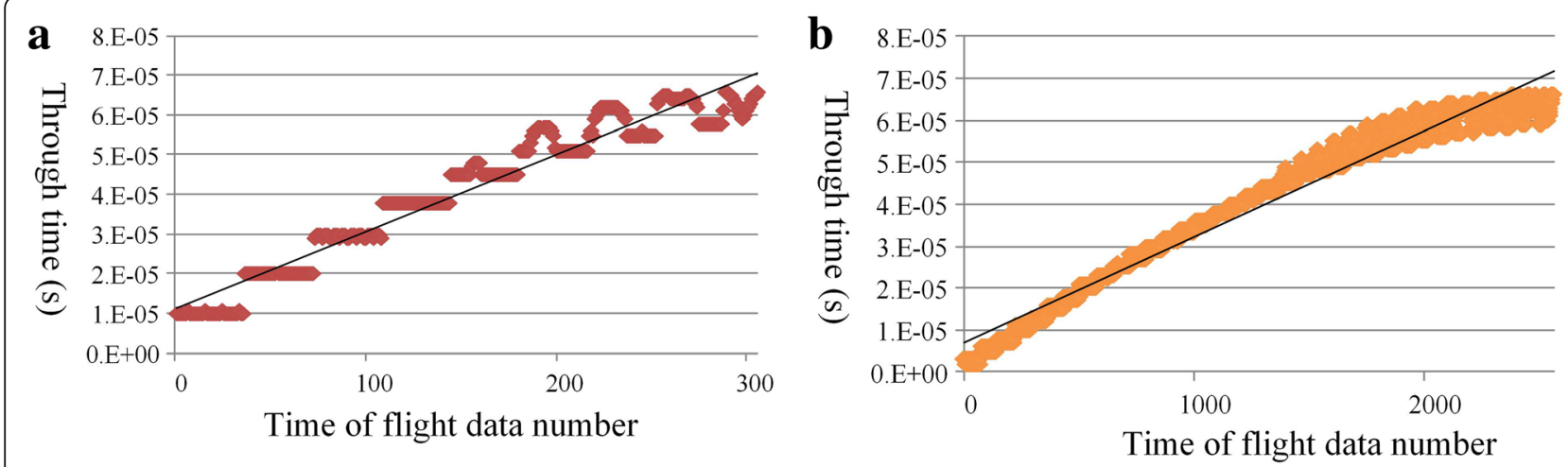

Fig. 5 Data distribution of measurement and interpolation: a distribution of 306 transit time data, b distribution of 2556 transit time data 

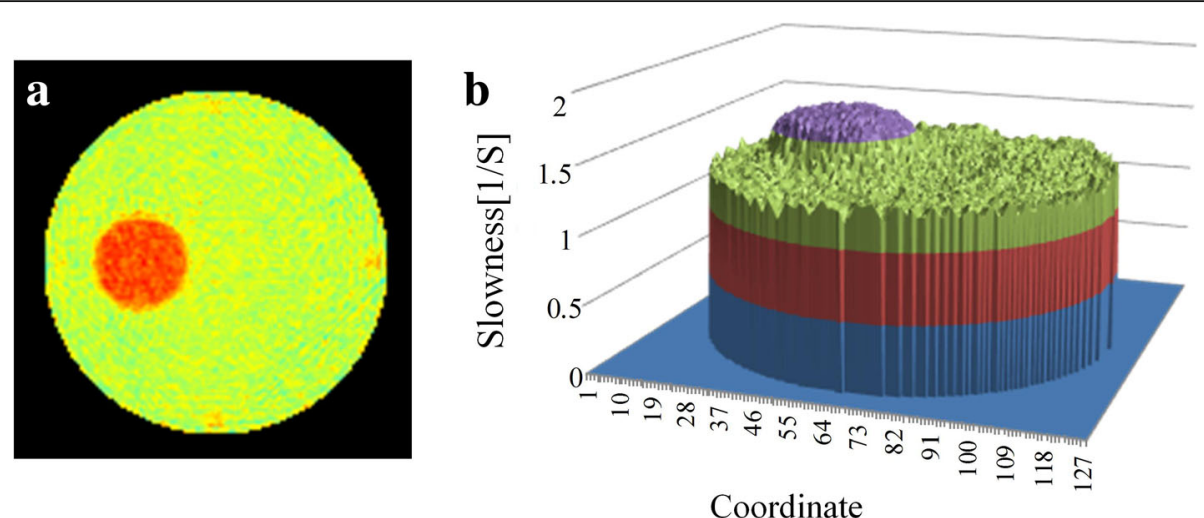

Fig. 6 Two thousand five hundred fifty-six data reconstruction image and slowness: a reconstruction image with 2556 data, b slowness of reconstruction image with 2556 data

and the velocity of the ultrasonic abnormal portion was set to $2500 \mathrm{~m} / \mathrm{s}$. The numerical phantoms were set uniform and internal defects which were composed of $128 \times 128$ pixels. The reconstruction image result of the healthy portion was shown in Fig. 3a. As the velocity of the ultrasonic internal structure of the data model was placed at unity, the reconstructed image was uniform. Slowness could show detailed reconstruction images, data, and specific information. Figure $3 \mathrm{~b}$ showed a healthy object slowness. The slowness values were basically the same. It initially proved the correctness of the image reconstruction system.

The number of parameters of the transit time decided the reconstruction image quality. First, the 306 transit time parameters were set in the numerical model, and based on the measurement interval, the data was divided into nine groups. The abnormal portion was set to the coordinates $x=40$ and $y=64$, and the radius $r$ was set to 15 units. As the transit time data were only 306 , the data distribution was not linear and continuous. The transit time parameters were laid together to the group for each fixed angle. For example, if the transit time parameters were measured with $20^{\circ}$ intervals, we can get from 20 to $180^{\circ}$ in nine groups.

In our simulation, the abnormal portion ultrasonic velocity was $2500 \mathrm{~m} / \mathrm{s}$. Since the measurement path goes through the defect position, so the five group transit time values in the group were not the same parameters. The transmission paths with abnormal portion ultrasonic velocities were higher than the healthy portion transmission paths.

The reconstruction image based on 306 transit time data and MLEM algorithm was shown in Fig. 4a. Although the defect could be detected in reconstruction images, only 306 transit time parameters were used. There were many artifacts, and the readability of the reconstructed image was relatively low. Figure $4 \mathrm{~b}$ shows the slowness of the reconstruction image with only 306 data. The defective part could be identified, but because the transit time parameters were too few, it interfered with the accuracy of image reconstruction.

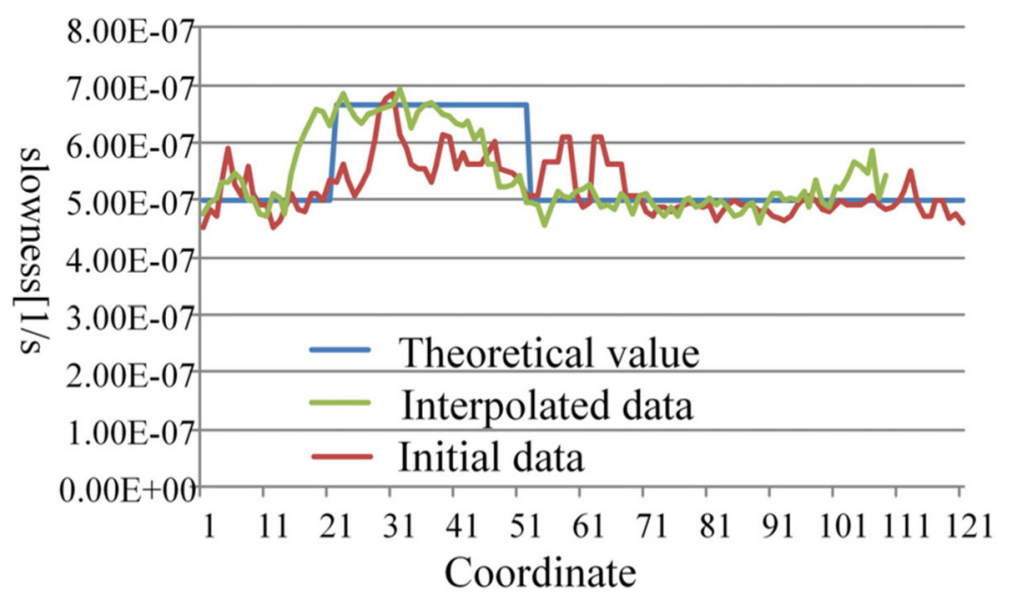

Fig. 7 The profile of distribution of reconstructed pixels 
Table 1 Parameters of concrete

\begin{tabular}{lllll}
\hline Material (unit, grams) & Water & Cement & Sand & Crushed stone \\
Amount & 1746 & 2691 & 7344 & 8982 \\
\hline
\end{tabular}

Under the 306 database, parallel and angle interpolation were used to get more transit time parameters. A number of path point of measurement became 35. The number of data interpolated angle increased to 612. Equal intervals of the measurement path distance were used to obtain the parallel beam. Figure 5b shows the 2556 transit time parameters after angle and parallel interpolation. All transit time parameters were continuously distributed. Compared with Fig. 5a, the data has been well amplified and has good clustering.

Based on the transit time parameter interpolation, a better quality reconstruction image could be reconstructed. The artifacts disappeared, and the defective portion could be clearly found in Fig. 6a. Slowness distribution could clearly reproduce the defect position and size as shown in Fig. 6b, which verified the effectiveness of the method of data interpolation and MLEM in ultrasonic image reconstruction system.

As a result, in order to apply the MLEM algorithm method of ultrasonic computed tomography, the transit time parameter interpolation was necessary. The distribution of reconstructed pixel values along the diameter in the images was plotted in Fig. 7. The profile of theoretical value, initial data, and interpolated data was shown.

\section{Concrete phantoms experimental results and discussions}

In our previous research, the image reconstruction of concrete members was based primarily on the FBP algorithm. In addition to the image reconstruction algorithm, the relevant experimental equipment such as data acquisition was the same. Defects ranging in diameter from 4 to $8 \mathrm{~cm}$ can be reconstructed by data difference processing suitable for the FBP algorithm. However, it is sometimes difficult to accurately locate the size and position of the defect in the reconstructed image of the concrete phantom. The other details could be found in our research paper [31].
A batch of concrete mixer and concrete materials were prepared to produce experimental concrete. The experimental materials prepared were water, cement, fine aggregate (sand), and coarse aggregate (crushed stone). They were stirred by a concrete mixer, and concrete phantom was formed by pouring this mix into a mold. The required amount was shown in Table 1 (unit, grams). To protect the quality of the concrete, the concrete production process was strictly observed when making the concrete phantoms. The strength of the concrete was at an ordinary level, and the water to cement ratio was $65 \%$.

In order to verify the proposed solution, we designed and poured four concrete models. Four pieces of concrete phantoms were prepared as test specimens as shown in Fig. 8. The diameter of the cylindrical concrete sample size was $10 \mathrm{~cm}$, and its height was $20 \mathrm{~cm}$.

Phantom A was ordinary concrete. The uniform and defect-free concrete sample can verify the transmission of ultrasonic signals in the test system and can also serve as a benchmark for signal comparison in the same concrete propagation path. A steel was set in phantom B; the purpose of the test was to verify the change in the ultrasonic signal through the concrete sample containing the reinforcement and whether the rebar position could be reconstructed in the image reconstruction system. There were cracks in phantom C. After the concrete hardened, phantom $C$ was placed over the uniform load from the side by using the machine for a compression test. Moreover, in order to maintain the cracks, the concrete was connected by wire. The purpose of this sample is to verify the extent to which cracks affect ultrasound propagation and image reconstruction systems. Concrete phantom D had a defect which was set in the center. The purpose is to verify the detection effect of the proposed image reconstruction system and experimental scheme on the cavity. The shape has a variable length of $4 \mathrm{~cm}$ square.

Due to the possibility of the leakage of signal noise in this experiment, it was necessary to further increase the value of the threshold. The resonance frequency of the vibrator was covered in an ultrasonic velocity computerized
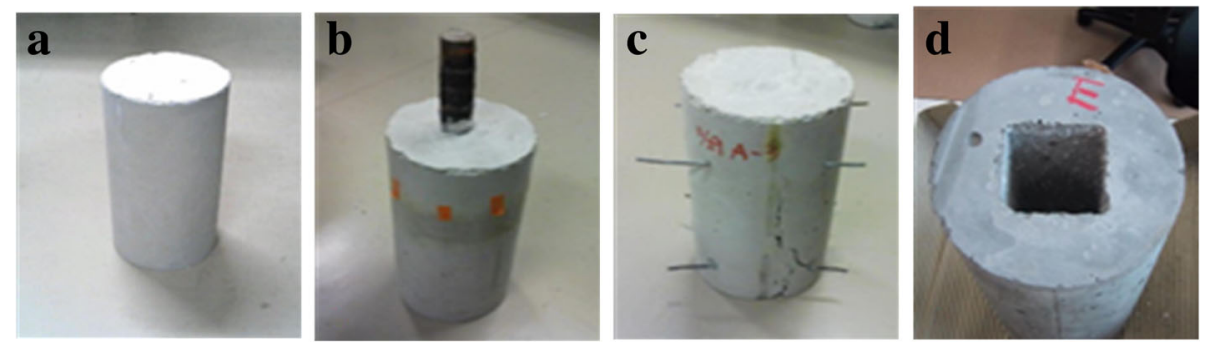

Fig. 8 Concrete phantoms 

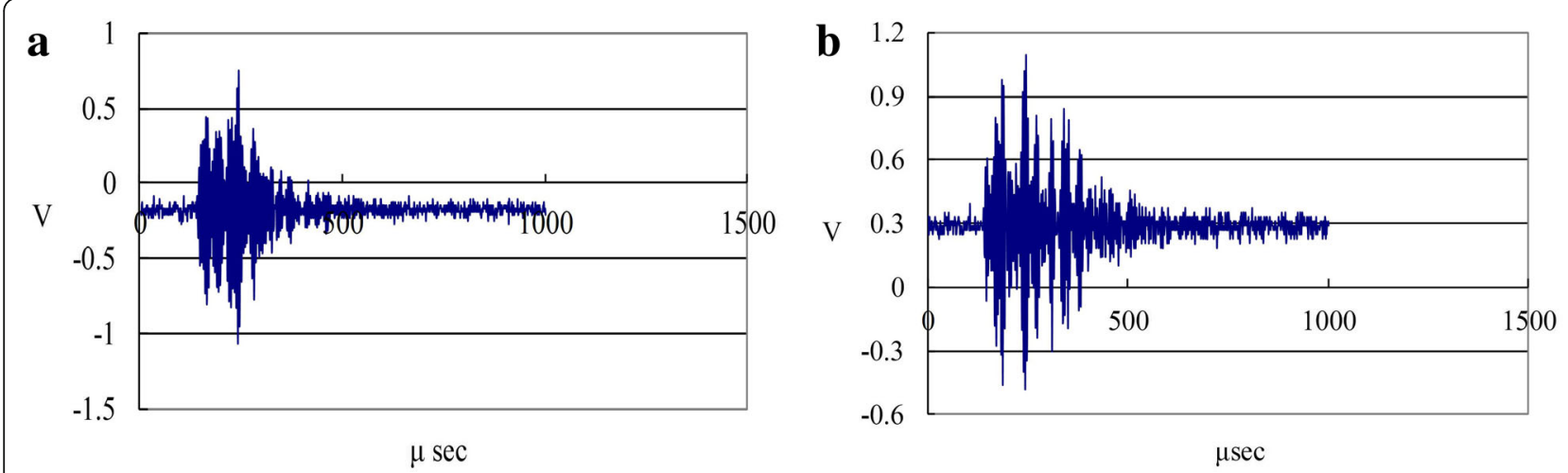

Fig. 9 Waveform data of concrete phantom; a waveform data of phantom A, b waveform data of phantom B

tomography apparatus around $63 \mathrm{kHz}$. The waveform data of phantom A and phantom B were measured in Fig. 9. As the internal structure of the concrete column was not the same, the measurement waveform was quite different.

The waveform data of concrete phantoms $\mathrm{A}$ and $\mathrm{B}$, as shown in Figs. 9a, b, could be calculated by transit time data. As there were cracks throughout the concrete phantom $\mathrm{C}$, we could not obtain the waveform signal. The point has been reached for the maximum amplitude of $10 \%$ as the transit time data, so the waveform data were processed based on a threshold.

The three images in Fig. 10 were the reconstruction results of the tests for concrete phantoms A, B, C, and D. From the reconstruction result, the concrete phantoms could be detected based on MLEM algorithm and interpolation data (Fig. 10a). The position and size of the steel could be found in reconstruction image Fig. 10b of phantom B, but it was possible that some artifacts appeared in the reconstruction image. The effective transit time parameters of concrete phantom $\mathrm{C}$ could not be calculated as they were affected by the cracks, so its image could not be reconstructed in Fig. 10c. The defect in concrete phantom $\mathrm{D}$ can be found in the reconstructed image of Fig. 10d. The defect position and size of the concrete phantoms were observed, but it was possible that some artifacts were recognized. The experimental results demonstrated the effectiveness of the proposed image reconstruction system.
Figure 11a shows the slowness of concrete phantom D. Although it could reconstruct the location and size of the defects, it was difficult to detect the shape of the defects because of the influence of ultrasound propagation characteristics. Figure 11b showed the distribution of the slowness values along the middle line in the reconstructed image. The interpolated data could reconstruct the defective portion more clearly than the original data.

The proposed scheme realizes the optimization of the transit time parameters and the image reconstruction of the concrete members. The system can reconstruct images of various types of concrete members, and the correctness of the experimental system has been verified. The number of transit time parameters has a significant impact on the accuracy of image reconstruction. Based on the data interpolation of angle and parallel, the quality of data image reconstruction after interpolation is significantly improved. This can solve the problem of a large amount of data required for image construction based on the ordered expectation maximum expected value iterative reconstruction algorithm and meet the practical engineering application of the measurement system.

\section{Conclusion}

In our simulation and concrete phantom experiment, the images were reconstructed by using transit time parameters interpolation and MLEM algorithm. The
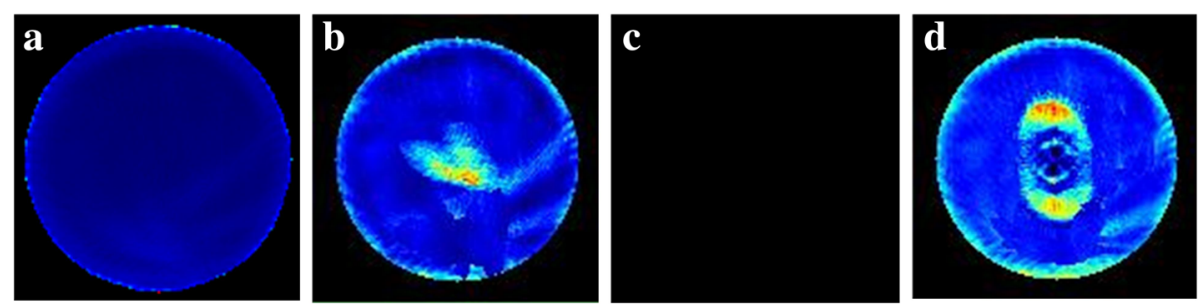

Fig. 10 Reconstruction images of concrete phantoms: a reconstrucion image of phantom $A, \mathbf{b}$ reconstrucion image of phantom $B$, c reconstrucion image of phantom $\mathrm{C}$, $\mathbf{d}$ reconstrucion image of phantom $\mathrm{D}$ 


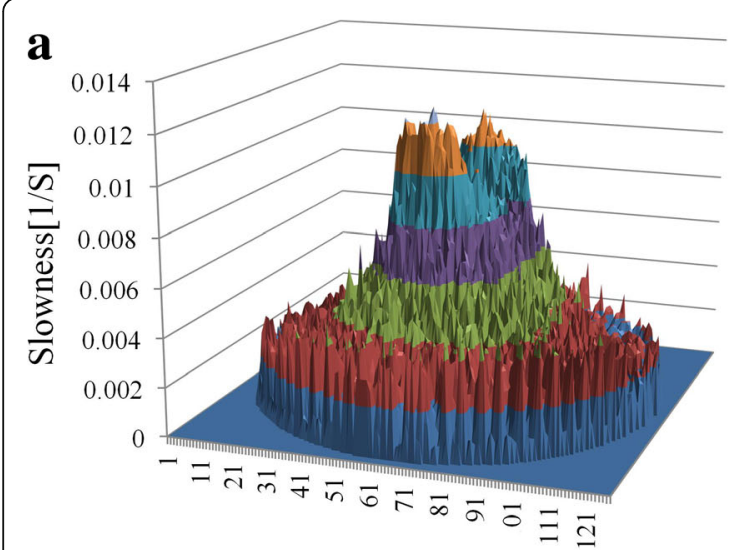

Coordinate

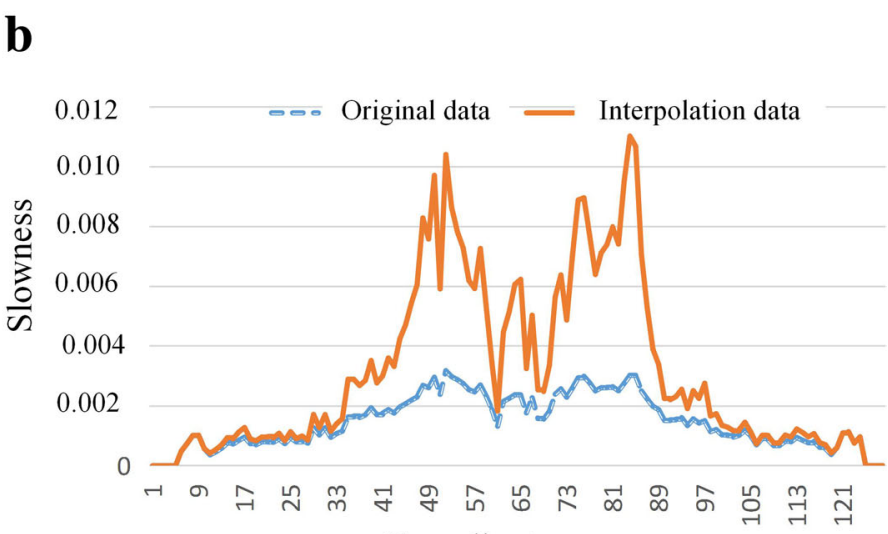

Coordinate

Fig. 11 Slowness of phantom D reconstruction image: a slowness of reconstruction image, b Slowness comarison of orininal data and interpolation data

propagation characteristics and detection signals of acoustic waves in the defective concrete pile foundation are analyzed. On this basis, the acoustic source frequency of acoustic sonic imaging and the selection and application of the observation system are studied.

The experimental results show that the image reconstruction can be performed on the abnormal parts of the speed of the concrete such as steel bars, voids, and steel pipes. Since the concrete gap seriously hinders the ultrasonic signal propagation, the TOF time parameter is not perfect, and the image reconstruction system based on the transit time parameters cannot perform image reconstruction on cracks.

The steel, defect position, and size could be formed in the image reconstruction results. The small shape of the defect was difficult to reconstruct in computerized tomography images, possibly because the diameter of the defect was smaller than the wavelength of the ultrasonic wave. In the image reconstruction results, we also found that, if the concrete cracks had a greater impact on the propagation of the ultrasound, it was difficult to obtain an image reconstruction. Although the defects were detected by the proposed method and the position and the size of the defect can be reconstructed in CT images, the shape of the defects was different from the actual defects. The possible reason for this was due to the anisotropic property of ultrasonic waves. Therefore, we should further research the impact of ultrasonic wave propagation on image reconstruction. The typical defect shapes considered are relatively regular, but the internal defects of concrete tend to be irregular, so the influence of irregular shape defects on sound wave propagation characteristics and defect position detection will be considered later.

The principle of image reconstruction system is to reproduce the sound velocity distribution of the target object based on the transit time parameter. The existing research results can quantitatively measure concrete, voids, and steel. Qualitative analysis and accurate image reconstruction of the above internal structures are important research directions in the future. However, our image reconstruction system still needs to be further improved for the prediction of defects, especially the defect shape of the system is different from the actual shape. This may be caused by the anisotropic characteristics of the ultrasound. So, further research on the effects of ultrasound propagation on image reconstruction is needed.

Abbreviation

MLEM: Maximum likelihood expectation maximization

\section{Acknowledgements}

The authors are very thankful to Tamura-Yanagida Laboratory and Atati Laboratory from the Yamagata University of Japan for providing the experimental data.

\section{Funding}

This work was supported by QingLan Project of Jiangsu Province and National Natural Science Fund of China (61806088).

\section{Availability of data and materials}

We can provide the data.

\section{About the authors}

Author 1-Honghui Fan, Doctor of Engineering, Associate Professor. Graduated from Yamgata University of Japan in 2011. Worked at the Jiangsu University of Technology. His current research interests include computer application technology, image processing, and image restoration.

Author 2-Hongjin Zhu, Doctor of Engineering, Associate Professor. Graduated from Yamgata University of Japan in 2010. Worked at the Jiangsu University of Technology. Her current research interests include image processing, computer vision, and pattern recognition.

\section{Authors' contributions}

Both authors took part in the discussion of the work described in this paper. $\mathrm{HF}$ designed the experiment and wrote the first version of the paper. $\mathrm{HZ}$ verified the proposed method and performed the part experiments of the paper. Both authors read and approved the final manuscript. 


\section{Competing interests}

Both authors declare that they have no competing interests. And both authors have seen the manuscript and approved to submit to your journal. We confirm that the content of the manuscript has not been published or submitted for publication elsewhere.

\section{Publisher's Note}

Springer Nature remains neutral with regard to jurisdictional claims in published maps and institutional affiliations.

Received: 27 August 2018 Accepted: 30 October 2018 Published online: 19 November 2018

\section{References}

1. R. Sloun, A. Pandharipande, M. Mischi, L. Demi, Compressed sensing for ultrasound computed tomography. IEEE Trans. Biomed. Eng. 62(6), 1660-1664 (2015)

2. B. Jiang, W. Zhao, W. Wang, Improved ultrasonic computerized tomography method for STS (steel tube slab) structure based on compressive sampling algorithm. Appl. Sci. 7(5), 432 (2017)

3. M. Pérezliva, J.L. Herraiz, J.M. Udías, E. Miller, B.T. Cox, B.E. Treeby, Time domain reconstruction of sound speed and attenuation in ultrasound computed tomography using full wave inversion. J. Acoust. Soc. Am. 141(3), 1595-1604 (2017)

4. A. Gajdacsi, A.J.C. Jarvis, P. Huthwaite, F.B. Cegla, Reconstruction of temperature distribution in a steel block using an ultrasonic sensor array. J. Nondestruct. Eval. 33(3), 458-470 (2014)

5. K. Wang, T. Matthews, F. Anis, C. Li, Waveform inversion with source encoding for breast sound speed reconstruction in ultrasound computed tomography. IEEE Transactions on Ultrasonics Ferroelectrics \& Frequency Control 62(3), 475-493 (2015)

6. W. Dong, Z. Wu, X. Zhou, Y. Tan, Experimental studies on void detection in concrete-filled steel tubes using ultrasound. Construction \& Building Materials 128, 154-162 (2016)

7. M. Simurda, L. Be, L. Duggen, N.T. Basse, A Fourier collocation approach for transit-time ultrasonic flowmeter under multi-phase flow conditions. J. Comput. Acoust. 25(4), 1750005 (2017)

8. H. Yanagida, Y. Tamura, K.M. Kim, J.J. Lee, Development of ultrasonic timeof-flight computed tomography for hard wood with anisotropic acoustic property. Jpn. J. Appl. Phys. 46(8A), 5321-5325 (2007)

9. J.D. Evans, D.G. Politte, B.R. Whiting, J.A. O'Sullivan, J.F. Williamson, Noiseresolution tradeoffs in $\mathrm{X}$-ray $\mathrm{CT}$ imaging: a comparison of penalized alternating minimization and filtered backprojection algorithms. Med. Phys. 38(38), 1444-1458 (2011)

10. A. Moscariello, R.A.P. Takx, U.J. Schoepf, M. Renker, P.L. Zwerner, T.X. O'Brien, T. Allmendinger, S. Vogt, B. Schmidt, G. Savino, C. Fink, L. Bonomo, T. Henzler, Coronary CT angiography: image quality, diagnostic accuracy, and potential for radiation dose reduction using a novel iterative image reconstruction technique-comparison with traditional filtered back projection. International Journal of Medical Radiology 21(10), 2130-2138 (2011)

11. H. Fan, H. Yanagida, Y. Tamura, S. Guo, T. Saitoh, T. Takahashi, Image quality improvement of ultrasonic computed tomography on the basis of MLEM algorithm considering anisotropic acoustic property and time-of-flight interpolation. Jpn. J. Appl. Phys. 49(7S), 07HC12 (2010)

12. K.C. Prabhat, M.K. Aditya, C. Phatak, C. Bouman, G.M. De, 3D reconstruction of the magnetic vector potential using model based iterative reconstruction. Ultramicroscopy 182, 131-144 (2017)

13. F. Hiroya, A. Kazunari, Y. Hirotaka, H. Tomoki, N. Tomoya, Improvement of the method for determination of time-of-flight of ultrasound in ultrasonic TOF CT. SICE Journal of Control, Measurement, and System Integration 10(6), 363-370 (2015)

14. G. Akamatsu, K. Ishikawa, K. Mitsumoto, T. Taniguchi, N. Ohya, S. Baba, K. Abe, M. Sasaki, Improvement in PET/CT image quality with a combination of point-spread function and time-of-flight in relation to reconstruction parameters. J. Nucl. Med. 53(11), 1716 (2012)

15. J.H. Jung, B.D. Shizgal, On the numerical convergence with the inverse polynomial reconstruction method for the resolution of the Gibbs phenomenon. J. Comput. Phys. 224(2), 477-488 (2007)
16. S.S. Chandra, I.D. Svalbe, J. Guédon, A.M. Kingston, N. Normand, Recovering missing slices of the discrete Fourier transform using ghosts. IEEE Trans. Image Process. 21(10), 4431-4441 (2012)

17. R. Muldoon, A. Chalker, M.C. Forde, M. Ohtsu, F. Kunisue, Identifying voids in plastic ducts in post-tensioning prestressed concrete members by resonant frequency of impact-echo, SIBIE and tomography. Constr. Build. Mater. 21(3), 527-537 (2007)

18. X. Liu, J. Wang, M. Yin, B. Edwards, P. Xu, Supervised learning of sparse context reconstruction coefficients for data representation and classification. Neural Comput. \& Applic. 28(1), 135-143 (2017)

19. S.-W. Jung, J.H. Park, Y.-S. Jeong, All-in-focus and multi-focus color image reconstruction from a database of color and depth image pairs. Multimedia Tools Appl. 75(23), 15493-15507 (2016)

20. M. Eguchi, H. Imasato, T. Yamakawa, Particle separation by employing nonuniform electric fields, traveling-wave electric fields and inclined gravity. Intelligent Automation \& Soft Computing 18(2), 121-137 (2012)

21. C. Yan, H. Xie, S. Liu, et al., Effective Uyghur language text detection in complex background images for traffic prompt identification. IEEE Trans. Intell. Transp. Syst. 19(1), 220-229 (2018)

22. C. Yan, H. Xie, J. Chen, Z. Zha, et al., An effective Uyghur text detector for complex background images. IEEE Transactions on Multimedia (2018). https://doi.org/10.1109/TMM.2018.2838320

23. P. Papaconstadopoulos, I.R. Levesque, R. Maglieri, J. Seuntjens, Direct reconstruction of the source intensity distribution of a clinical linear accelerator using a MLEM algorithm. Phys. Med. Biol. 61(3), 1078-1094 (2016)

24. M. Gonzalez, C. Minuesa, I. del Puerto, Maximum likelihood estimation and expectation-maximization algorithm for controlled branching processes. Computational Statistics \& Data Analysis 93, 209-227 (2016)

25. J. Nuyts, C. Michel, P. Dupont, Maximum-likelihood expectation-maximization reconstruction of sinograms with arbitrary noise distribution using NECtransformations. IEEE Trans. Med. Imaging 20(5), 365-375 (2001)

26. H. Fan, H. Zhu, X. Zhao, J. Zhang, D. Wu, Q. Han, Ultrasonic image reconstruction based on MLEM for concrete structural information. Computers \& Electrical Engineering 62, 293-301 (2017)

27. D. Aloni, A. Stern, B. Javidi, Three-dimensional photon counting integral imaging reconstruction using penalized MLEM. Opt. Express 19(20), 19681-19687 (2011)

28. A. Mehranian, H. Zaidi, A.J. Reader, MR-guided joint reconstruction of activity and attenuation in brain PET-MR. Neuroimage 162, 276-288 (2017)

29. G.B. Wang, J.Y. Qi, Penalized likelihood PET image reconstruction using patch-based edge-preserving regularization. IEEE Trans. Med. Imaging 31(12), 2194-2204 (2012)

30. W. Ye, K.K. Ma, Color image demosaicing using iterative residual interpolation IEEE Trans. Image Process. 24(12), 5879-5891 (2015)

31. H. Fan, H. Zhu, Q. Han, Image reconstruction of concrete based on filtered backprojection method using ultrasonic time of flight data. Computer Modelling and New Technologies 18(6), 72-78 (2014)

\section{Submit your manuscript to a SpringerOpen ${ }^{\bullet}$ journal and benefit from:}

- Convenient online submission

- Rigorous peer review

- Open access: articles freely available online

- High visibility within the field

- Retaining the copyright to your article

Submit your next manuscript at $>$ springeropen.com 